\title{
Efectos de la suplementación con vitamina $D$ en el control del asma en pacientes adultos: una revisión sistemática
}

\author{
DEMIAN MARTÍNEZ P.*, MAYLINE P. VILCHES A.*, FRANCISCO MALDONADO G.*, \\ LUCAS CARTES S.* y JAVIER ROJAS V.******
}

\section{Effects of vitamin D supplementation on asthma control in adult patients: a systematic review}

Background: Asthma is an airway chronic disease, with an important inflammatory component within its pathogenesis, driven by a dysregulated immune response. Vitamin D is an immunomodulator that regulates cell proliferation, differentiation and cytokine secretion profile. An association between vitamin D sufficiency and improvement in pulmonary function, asthma control and a decrease in exacerbations have been proposed in the adult population, which falls into importance given the high prevalence of vitamin D insufficiency globally. Objective: To know vitamin D supplementation effects in asthma control in adults. Methods: Through a PubMed and EMBASE database search, a systematic review of the literature was conducted. Primary outcomes were: changes in FEV $V_{1}$, symptomatic control, exacerbation frequency and PEF and adverse events as secondary outcomes. Outcome evidence quality assessment was made using the GRADE model. Results: Seven studies were selected after taking out duplicates, applying inclusion and exclusion criteria. In all cases, evidence quality assessed by the GRADE system yielded very low quality. Conclusions: No statistically significant differences were found after vitamin D supplementation in the overall evaluated outcomes. Nonetheless, a cautious interpretation of studies is mandatory, because evidence quality was very low and no serious adverse events were reported. Hence this treatment usefulness as an ancillary therapy for vitamin $D$ deficient asthmatic patients cannot be dismissed.

Key words: Asthma; Adults; Vitamin D; Exacerbations; Lung Function; Asthma Control; Systematic Review.

\section{Resumen}

Introducción: El asma es una enfermedad crónica inflamatoria de la vía aérea e inmunomediada en su patogénesis. La vitamina D es un inmunomodulador que regula el perfil secretor de citoquinas, entre otras funciones celulares. Una asociación entre la suficiencia de vitamina D y mejoría en la función pulmonar, control de asma y número de exacerbaciones se ha propuesto en adultos, importante dada la elevada prevalencia de insuficiencia de vitamina D globalmente. Objetivo: Conocer los efectos de la suplementación con vitamina D en el control del asma en adultos. Métodos: Se realizó una revisión sistemática de la literatura a través de una búsqueda en la base de datos PubMed y EMBASE. Los desenlaces primarios fueron cambios en $V E F_{1}$, control sintomático, frecuencia de exacerbaciones, además de eventos adversos y FEM como desenlaces secundarios. La calidad de evidencia de los desenlaces fue evaluada a través del modelo GRADE. Resultados: Siete estudios fueron seleccionados después de remover duplicados y aplicar los criterios de inclusión y exclusión, con calidad de evidencia muy baja aplicando sistema GRADE. Discusión: No se encontraron diferencias estadísticamente significativas tras la suplementación con vitamina $D$ en los desenlaces evaluados en general, pero dada

\footnotetext{
* Interno/a de Medicina, Facultad de Medicina Universidad de Chile. Santiago, Chile.

** Médico Internista. Hospital San Juan de Dios. Santiago, Chile.

***Residente Unidad Paciente Crítico. Hospital San Juan de Dios. Santiago, Chile.
} 
la calidad de evidencia muy baja y que no se reportaron efectos adversos serios, es necesario tomar cautelosamente estos resultados. Asímismo no se puede descartar la utilidad de esta terapia como tratamiento auxiliar a los pacientes asmáticos con este déficit vitamínico.

Palabras clave: Asma; Adultos; Vitamina D; Exacerbaciones; Función pulmonar; Control del Asma; Revisión Sistemática.

\section{Introducción}

El asma es un trastorno crónico de la vía aérea que afecta a gran parte de la población mundial, constituyendo un problema mayor de salud pública y una considerable carga económica para los sistemas sanitarios ${ }^{1,2}$.

La obstrucción reversible e hipersensibilidad de la vía aérea, sumado a los procesos de inflamación crónica e infiltración celular, constituyen sus características principales. Estas determinan su clínica obstructiva recurrente y fluctuante en intensidad, que además se asocia a limitación del flujo aéreo espiratorio ${ }^{3}$. Posee una etiología multifactorial, comprendiendo interacciones entre susceptibilidad genética, factores individuales y exposición ambiental.

El sistema inmune innato y adaptativo participan en la patogénesis del asma, vía complejos mecanismos que culminan en una respuesta inmune desregulada ${ }^{4}$. En el asma atópica, posterior a la exposición a un alergeno, existiría una sobrerregulación de la respuesta inflamatoria mediada por células T-helper tipo 2 (Th2), con liberación de citoquinas proinflamatorias como Interleuquina (IL)-4, IL-5, IL-9 e IL-13 ${ }^{5}$, determinando funciones como el reclutamiento de eosinófilos, degranulación mastocitaria, producción de inmunoglobulina $\mathrm{E}$ ( $\mathrm{IgE}$ ), hiperplasia de las células caliciformes, hipersecreción de mucus e hipersensibilidad de la vía aérea ${ }^{6}$. Actualmente, dado el mayor conocimiento de la fisiopatología del asma, se reconoce también el rol patogénico de la disfunción epitelial y de la inmunidad innata ${ }^{6,7}$.

\section{Vitamina D y su rol inmunomodulador}

La vitamina D (VD) es una vitamina liposoluble obtenida con la dieta o vía síntesis endógena mediada por la exposición cutánea a radiación solar. Se metaboliza en el hígado y luego en el riñón para obtener su metabolito activo 1,25 -dihidroxi-VD, también conocido como calcitriol, el que interactúa a nivel intracelular con su receptor Vitamin D Receptor, regulando la transcripción de numerosos genes. Recientemente se ha posicionado el potencial rol inmunomodulador de la $\mathrm{VD}^{8}$, evidenciable a través de la presencia de receptores para esta en células del sistema inmune. A nivel de la inmunidad innata, disminuye la secreción de citoquinas proinflamatorias, promueve la secreción de citoquinas antiinflamatorias a nivel mastocitario y disminuye la capacidad dendrítica de madurar y activar células $\mathrm{T}$. Respecto a la inmunidad adaptativa, se asociaría con un cambio de perfil de secreción de citoquinas de los linfocitos Th2, supresión de la respuesta Th1 y Th17, disminución de la proliferación de linfocitos $\mathrm{B}$ y producción de anticuerpos, conduciendo finalmente a un escenario antiinflamatorio ${ }^{9}$.

Específicamente, respecto a la inflamación de la vía aérea, estudios en células animales y humanas han demostrado la interacción de la VD con células inmunes, células epiteliales y del músculo liso a nivel bronquial, reduciendo la secreción de IgE y la población de eosinófilos, generando una disminución de la inflamación, hipersensibilidad y remodelación de la vía aérea ${ }^{10-12}$.

\section{Vitamina D y Asma}

El déficit de VD se define como la concentración sérica de $25(\mathrm{OH}) \mathrm{D}$ menor a $20 \mathrm{ng} / \mathrm{ml}$ $(50 \mathrm{nmol} / \mathrm{L})$, mientras que niveles insuficientes corresponden al rango entre 21-29 ng/ml (50-70 $\mathrm{nmol} / \mathrm{L})^{13}$. Sin embargo, no existe consenso respecto a los valores óptimos. Ambas condiciones son altamente prevalentes, explicado por factores como: escasa exposición solar, uso de fotoprotección, aumento de actividades puertas adentro y cambios dietarios ${ }^{12}$.

Extrapolando lo anterior a individuos asmáticos, es posible inferir que una fracción importante de estos presenta niveles insuficientes/deficientes de VD, lo que sumado a sus funciones inmunomoduladoras, ha suscitado la investigación en este campo. Actualmente, existe evidencia contradictoria respecto a la relación entre hipovitaminosis $\mathrm{D}$, el desarrollo de asma y complicaciones derivadas de ella en población adulta y pediátrica ${ }^{14}$. En niños algunos estudios han encontrado una asociación entre niveles deficientes de VD y mayor tasa de exacerbaciones ${ }^{15}$, mientras que en población adulta la evidencia es inconsistente ${ }^{16,17}$. Esto confirma el debate actual respecto al real beneficio de la suplementación con VD como terapia adyuvante en el manejo del asma en población adulta, fomentando la necesidad de estudiar y esclarecer su función, considerando su rol inmunomodulador, el am- 
plio acceso y bajo costo de las formulaciones farmacológicas disponibles, la efectividad en lograr niveles séricos fijados como objetivo y la seguridad del tratamiento

\section{Objetivo}

Conocer la eficacia de la suplementación con vitamina $\mathrm{D}$ en el control del asma en la población adulta.

\section{Metodología}

\section{Búsqueda sistemática de bibliografía}

Como directrices, se utilizaron las recomendaciones Preferred Reporting Items for Systematic Reviews and Meta-Analyses (PRISMA) (Figura 1). Durante julio de 2020 se realizó una búsqueda sistemática de bibliografía en las bases de datos PubMed y EMBASE, seleccionando estudios publicados entre los años 2014-2020. Con los operadores " $A N D$ " para conectores y "OR" para sinónimos o desenlaces y basándose en el sistema Patient, Intervention, Comparator/Control, Outcome (PICO), se utilizaron términos del vocabulario terminológico Medical Subject Headings (MeSH): "Asthma" AND "Adult" AND "Vitamin D" OR "Cholecalciferol" OR "ergocalciferol" AND "forced expiratory volume" OR "peak expiratory flow rate" OR "symptom flare up". En la variable resultado se utilizaron palabras libres: "FEV (1)" OR "PEF" OR "Clinical status" OR “exacerbation”. El tipo de publicación se limitó a ensayos clínicos randomizados (ECR) en idioma inglés.

\section{Selección de estudios}

Se definieron criterios de inclusión:

1) ECR.

2) Adultos con diagnóstico de asma.

3) Intervención con VD o análogos (sin importar vía de administración, posología o que se haya acompañado de otros fármacos para el tratamiento del asma) con monitorización de los niveles séricos de VD antes y después de la intervención.

Citaciones incluidas a partir de la búsqueda electrónica $(n=186)$

- $P U B M E D=93$ (Búsqueda realizada el 04 de Julio de 2020)

- $\mathrm{EMBASE}=93$ (Búsqueda realizada el 04 de Julio de 2020)

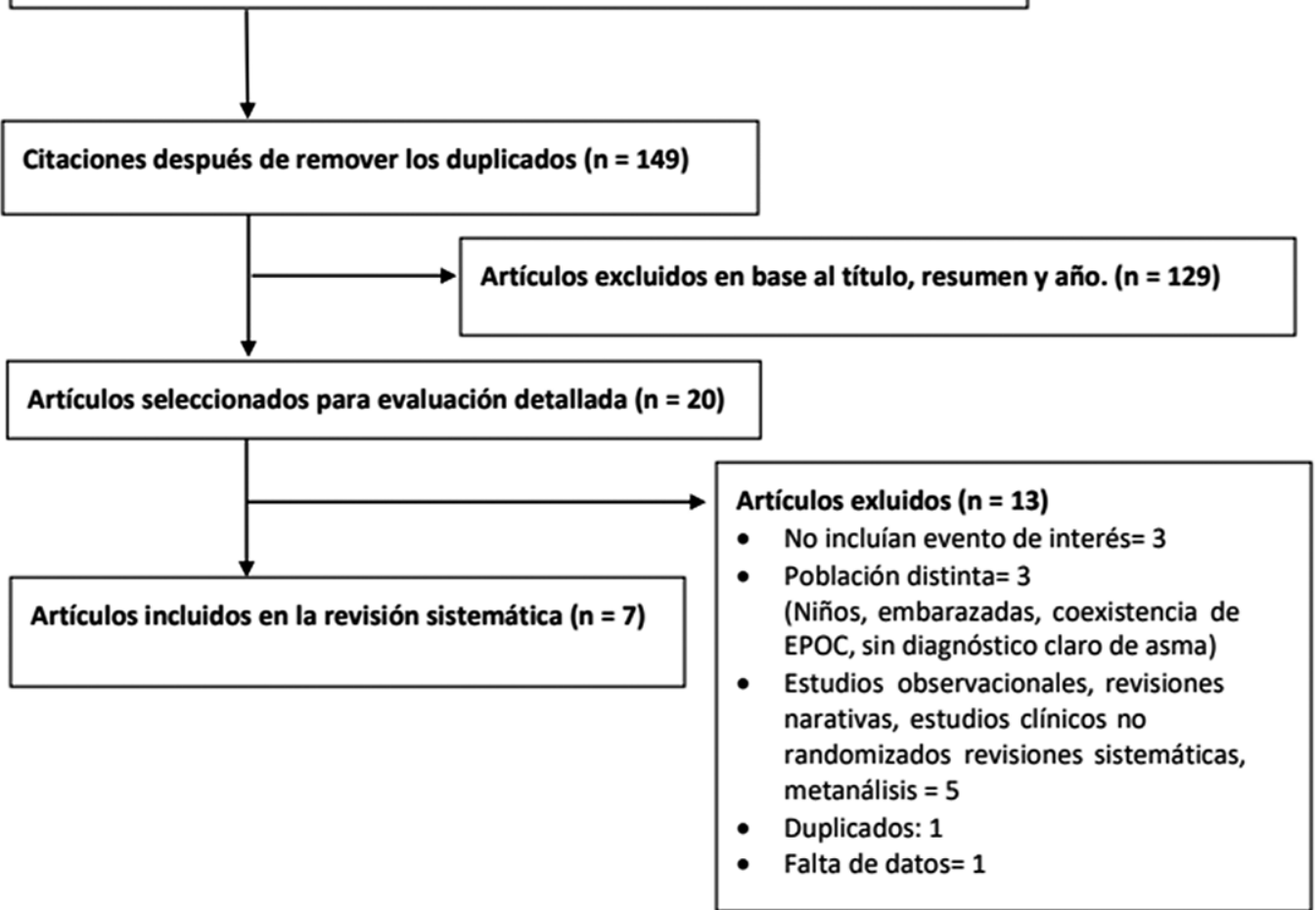

Figura 1. Flujograma para la selección de los estudios utilizados. 
4) Cumplir con alguno de los siguientes desenlaces: volumen espiratorio forzado en el primer segundo $\left(\mathrm{VEF}_{1}\right)$, control de los síntomas del asma mediante cuestionarios estandarizados o la frecuencia/presencia de exacerbaciones.

Como criterios de exclusión se usó:

1) Estudios no ECR.

2) Población embarazada o pediátrica (menor de 14 años).

3) Diagnóstico de enfermedad pulmonar obstructiva crónica (EPOC).

4) Suplementación con VD sin monitorizar niveles séricos pre y post intervención.

5) Estudios no finalizados.

6) Estudios que no incluyeron ninguno de los eventos de interés.

\section{Desenlaces}

Los desenlaces utilizados en los criterios de inclusión derivan de la definición de "control del asma” según la Guía Clínica Global Initiative for Asthma 2020, en la cual se integran dos dominios: control sintomático y riesgo futuro de resultados clínicos adversos ${ }^{3}$.

Para valores espirométricos, se consideró la variación del $\mathrm{VEF}_{1}$ respecto a su basal, tanto porcentual como volumétricamente. Para el control sintomático, se incluyó la valoración de síntomas mediante cuestionarios estandarizados: Asthma Control Test (ACT), Asthma Symptom Utility Index (ASUI) y Asthma Control Questionnaire (ACQ), considerándose la variación de su puntaje respecto al obtenido basalmente. Dada la heterogeneidad de definiciones de exacerbación, el grupo investigador la caracterizó como el empeoramiento agudo de síntomas/signos atribuibles al asma que: haya sido evaluada por un profesional médico, haya requerido ajuste de terapia de mantención o presente falla en el tratamiento de rescate, manifieste un empeoramiento agudo de su valoración espirométrica, haya requerido corticoterapia sistémica, o bien, haya requerido hospitalización o consulta en Servicio de Urgencias. Para esta variable, se consideró el tiempo hasta la primera exacerbación, el porcentaje de pacientes que presentaron una primera exacerbación en un tiempo dado y la tasa de exacerbaciones (número de eventos por persona-año).

Como desenlaces secundarios, se consideraron la variación del flujo espiratorio máximo (FEM) en litros/min respecto a su basal y los eventos adversos, evaluados en número de eventos reportados.

\section{Extracción de datos}

Para elaborar conclusiones y facilitar la com- paración entre estudios, se esquematizó la información (Tablas 1 y 2).

\section{Evaluación de la calidad}

La calidad de cada estudio fue evaluada por dos autores con las herramientas Cochrane de riesgo de sesgos respecto a los sesgos expresados en la Figura 2. Para evaluar la calidad de la evidencia respecto a los desenlaces, se utilizó el modelo Grading of Recommendations, Assessment, Development and Evaluation (GRADE) a través del software GRADEpro ${ }^{\circledR}$. Tres revisores fueron asignados para evaluar la calidad de los estudios en base a los criterios expresados en la Tabla 3.

\section{Resultados}

\section{Selección de estudios}

Se encontraron 186 citaciones, de los cuales se seleccionaron finalmente siete artículos ${ }^{18-24}$ para la revisión (Figura 1), luego de eliminar duplicados y de una revisión en dos etapas.

\section{Características de los estudios}

Las principales características se resumen en la Tabla 1 y Tabla 2. Esta revisión abarca un total de 965 pacientes asmáticos, con predominancia femenina en cuatro estudios ${ }^{19,20,23,24}$ y masculina en dos estudios ${ }^{18,21}$. En la descripción del perfil asmático de los pacientes, destaca el reclutamiento de asmáticos no atópicos en uno de ellos ${ }^{22}$. Los esquemas de intervención con VD también difieren: seis ${ }^{18,20-24}$ utilizan esquemas de colecalciferol, uno en formulación intramuscular ${ }^{18}$ y cinco administrados por vía oral ${ }^{20-24}$; mientras que un estudio ${ }^{19}$ utiliza alfacalcidol, derivado sintético de la VD. La intervención fue evaluada como terapia adyuvante en todos los estudios, definiéndose en tres ${ }^{19,21,24}$ un tratamiento específico, mientras que el resto ${ }^{18,20,22,23}$ utilizó un tratamiento de control inespecífico guiado por la severidad del asma. Respecto al estado de VD, todos los estudios incluyen población con niveles deficientes. El rango de seguimiento fue desde nueve semanas a doce meses, cuatro estudios ${ }^{18,20-22}$ con un seguimiento menor o igual a tres meses y sólo un estudio $^{23}$ con seguimiento de doce meses.

\section{Evaluación de la calidad}

La evaluación del riesgo de sesgo se resume en la Figura 2. Respecto al sesgo de selección, cuatro estudios reportaron generación de secuencia al azar ${ }^{18,21,23,24}$, siendo calificados con bajo riesgo, dos estudios no explicitaron el mé- 


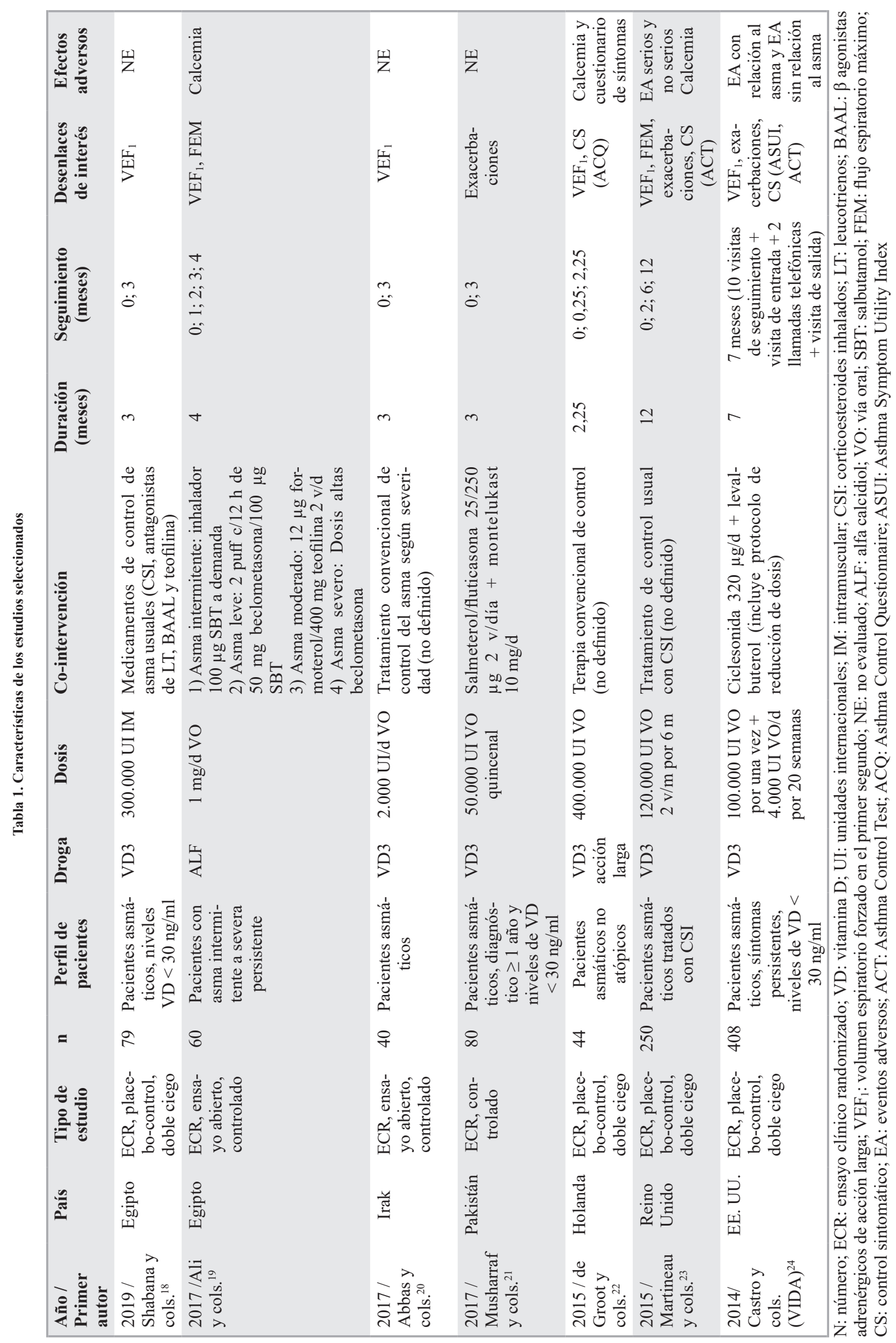




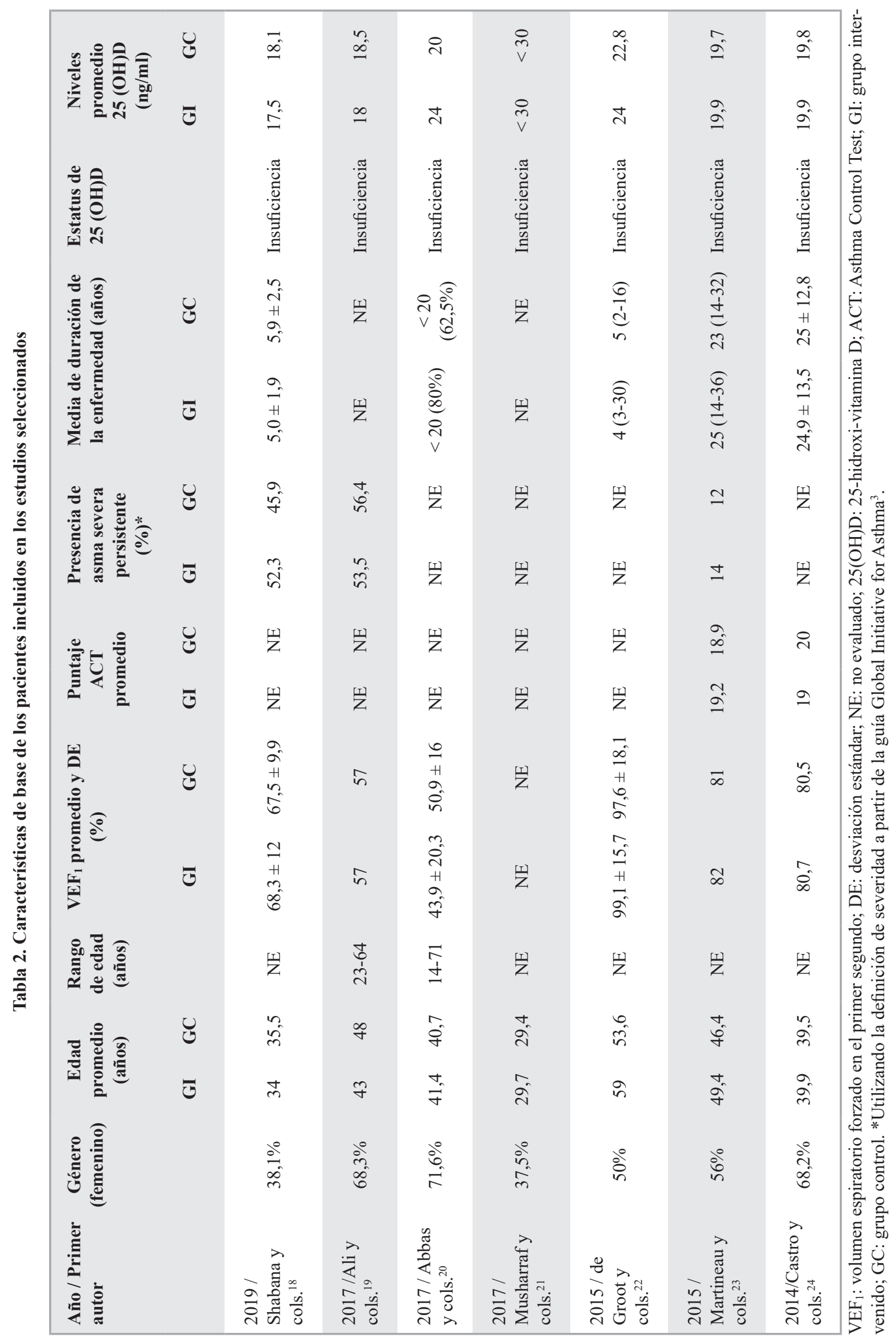




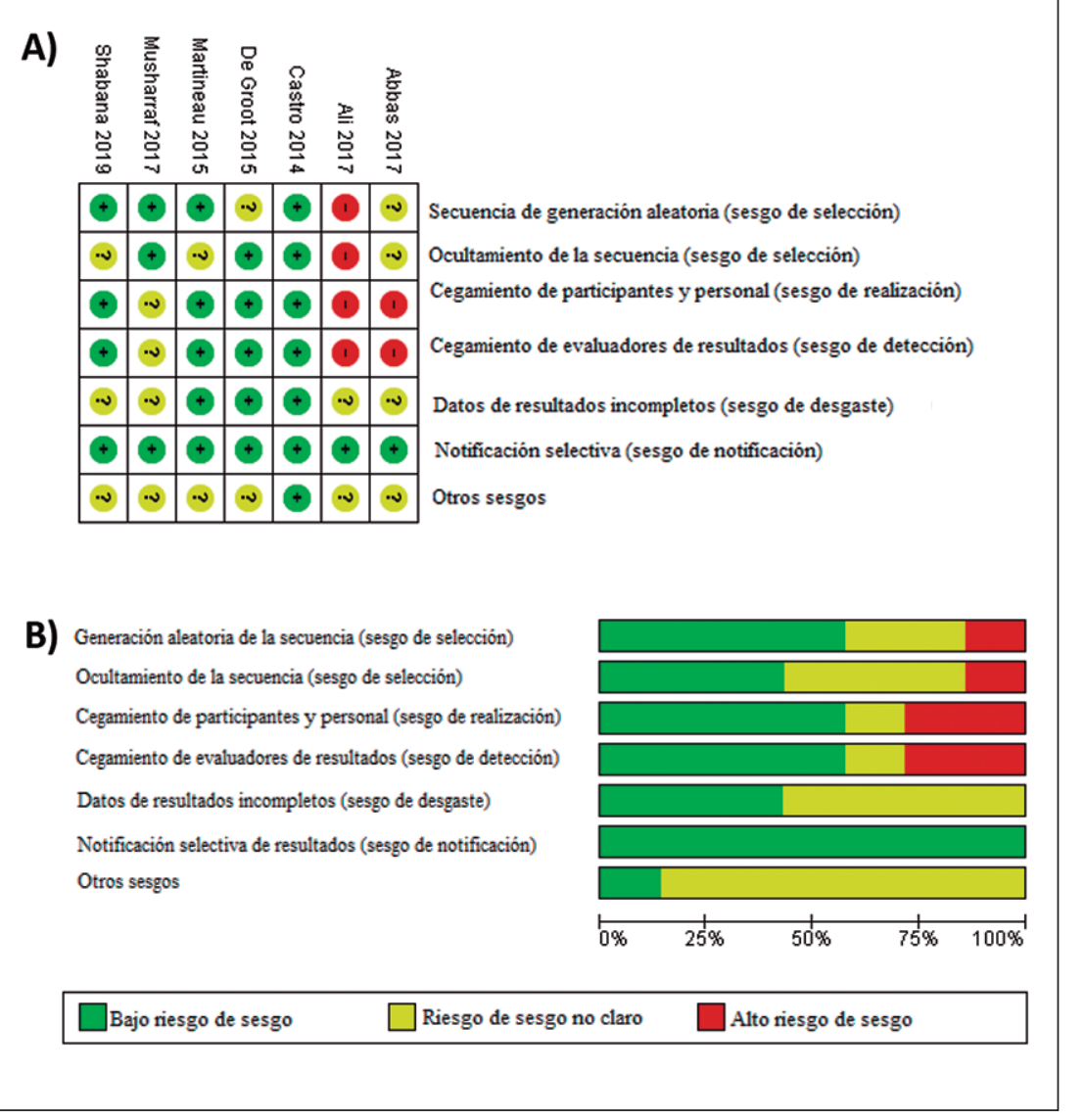

Figura 2. Evaluación de riesgo de sesgo de los estudios incluidos en la revisión según directrices Cochrane. $A$. Caracterización de riesgo de sesgo de cada estudio individual. $B$. Estimación porcentual del riesgo de sesgo total de los estudios seleccionados. todo de generación de secuencia aleatoria ${ }^{20,22}$, siendo calificados con riesgo no claro. Sólo un estudio fue calificado con alto riesgo al utilizar un método cuasi-aleatorio ${ }^{19}$. En relación a ocultamiento de la secuencia, tres estudios la reportaron, siendo calificados como de bajo riesgo $^{21,22,24}$; otros tres estudios fueron calificados como riesgo no claro dado que no se estableció si la intervención se entregó en un formato oculto $^{18,20,23}$. Sólo un estudio se consideró de alto riesgo de sesgo dado que entregó una píldora extra al grupo intervenido.

Respecto al cegamiento de los participantes y el personal y la evaluación de resultados, dos estudios notificaron ser de tipo ensayo abierto ${ }^{19,20}$, por lo que fueron calificados de alto riesgo de sesgo. De los cinco estudios restantes, cuatro informaron ser tipo doble-ciego ${ }^{18,22-24}$, por lo que fueron considerados de bajo riesgo; mientras que uno calificó como riesgo de sesgo no claro ${ }^{21}$. En datos de resultados incompletos, cuatro estudios calificaron con un riesgo de sesgo no claro $^{18-21}$, pues no explicitan la causa de pérdida de pacientes. En la categoría de reporte selectivo, todos los estudios tuvieron bajo riesgo de sesgo, dado que en sus resultados expresaron variables que estaban estipuladas previamente en su metodología. No se detectaron otros sesgos.

\section{Evaluación de desenlaces}

La calidad de evidencia tras la evaluación con el sistema GRADE (Tabla 3), resultó ser de muy baja calidad debido al alto riesgo de sesgo, imprecisión e inconsistencia. Respecto a esta última, fue calificada como "seria" ante la presencia de discordancia entre los resultados de los estudios para un mismo desenlace. La imprecisión de los resultados fue calificada como "seria" para todos los desenlaces evaluados, puesto que la revisión no supera el tamaño muestral de pacientes mínimos definido según "Optimal Information Size" (OIS) para el estudio más adecuado según poder, el cual fue calculado por Castro y cols. ${ }^{24}$, usando el programa "PS Power and Sample Size ${ }^{\circledR}$ ". La evidencia indirecta fue calificada como "no seria" para los desenlaces de función pulmonar $\mathrm{y}$ exacerbaciones, dado que todos los estudios evaluaron desenlaces que responden directamente 
Tabla 3. Evaluación GRADE de la calidad de la evidencia de los desenlaces

\begin{tabular}{|lccccccc|}
\hline Desenlace & $\begin{array}{c}\text { n de } \\
\text { estudios }\end{array}$ & $\begin{array}{c}\text { Diseño de } \\
\text { estudio }\end{array}$ & $\begin{array}{c}\text { Riesgo de } \\
\text { sesgo }\end{array}$ & Inconsistencia & $\begin{array}{c}\text { Evidencia } \\
\text { indirecta }\end{array}$ & Imprecisión & $\begin{array}{c}\text { Calidad de } \\
\text { la evidencia }\end{array}$ \\
$\mathrm{VEF}_{1}$ & 6 & $\mathrm{ECR}$ & Serio $^{\mathrm{a}}$ & Serio $^{\mathrm{b}}$ & No es serio & Serio $^{\mathrm{c}}$ & Muy baja \\
Exacerbaciones & 3 & $\mathrm{ECR}$ & Serio $^{\mathrm{a}}$ & Serio $^{\mathrm{b}}$ & No es serio & Serio $^{\mathrm{c}}$ & Muy baja \\
$\begin{array}{l}\text { Scores de control } \\
\text { sintomático }\end{array}$ & 3 & $\mathrm{ECR}$ & Serio $^{\mathrm{a}}$ & No es serio $^{\mathrm{b}}$ & Serio & Serio $^{\mathrm{c}}$ & Muy baja \\
\hline
\end{tabular}

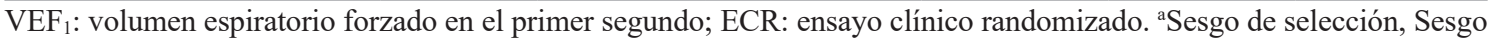
de realización, Sesgo de detección, Sesgo de desgaste y sesgo de notificación fueron los sesgos incluidos dentro de esta categoría. ${ }^{b}$ Se reportó inconsistencia por moderada o alta heterogeneidad en los resultados. ${ }^{\mathrm{E}} \mathrm{E}$ tamaño total de la muestra es menor que el TOI (tamaño óptimo de información), calificándose como impreciso.

a la pregunta de interés planteada y compararon de manera directa la intervención respecto a una población control. Por otro lado, para el control sintomático se consideró la evidencia indirecta como "seria" porque agrupa cuestionarios distintos entre sí como herramientas de evaluación.

La valoración estadística de los desenlaces evaluados se presenta en la Tabla 4.

\section{Función pulmonar}

Seis estudios evaluaron la función respiratoria $^{18-20,22-24}$. La calidad de este desenlace según el sistema GRADE resultó ser muy baja (Tabla 3). Dos estudios encontraron una mejoría significativa en el $\mathrm{VEF}_{1}$ entre el grupo intervenido con VD respecto al grupo control, el resto no mostró diferencias significativas respecto al $\mathrm{VEF}_{1}{ }^{20,22-24}$.

En relación al FEM, este fue evaluado en dos estudios ${ }^{19,23}$, ambos de tipo doble ciego y placebo-control. No se observaron diferencias significativas al comparar entre los grupos intervenido y control.

\section{Exacerbaciones}

Las exacerbaciones fueron evaluadas en tres de los siete estudios ${ }^{21,23,24}$. La calidad del desenlace se definió como muy baja (Tabla 3). En $\operatorname{dos}^{23,24}$ estudios no se hallaron diferencias estadísticamente significativas respecto a exacerbaciones al comparar entre el grupo tratado con VD y el grupo control, abarcando estos estudios la mayor parte de la población estudiada para este desenlace. El tercer estudio ${ }^{21}$ demostró una disminución significativa en el número de exacerbaciones. En el estudio VIDA ${ }^{24}$ se evidenció una disminución estadísticamente significativa en la tasa de exacerbaciones y en el tiempo hasta la primera exacerbación en el subgrupo de pacientes tratados con VD que alcanzó niveles de suficiencia al final del seguimiento respecto al grupo control.

\section{Control sintomático}

El control sintomático fue evaluado por tres estudios $^{22-24}$, todos doble ciego y controlados con placebo. Uno consideró cambios en el puntaje del cuestionario $\mathrm{ACQ}^{22}$, otro en el cuestionario $\mathrm{ACT}^{23}$ y el último en cuestionarios ACT/ASUI ${ }^{24}$. Se definió la calidad de la evidencia como muy baja según evaluación GRADE (Tabla 3). En ninguno de los estudios mencionados se hallaron cambios estadísticamente significativos en el puntaje obtenido en los cuestionarios $\mathrm{ACQ}^{22}, \mathrm{ACT}^{23,24}$ y $\mathrm{ASUI}^{24}$ al comparar el grupo intervenido y el grupo control.

\section{Eventos adversos}

Cuatro estudios evaluaron la ocurrencia de eventos adversos ${ }^{19,22-24}$. Tres estudios ${ }^{19,22,23}$ evaluaron el nivel de calcemia, sin diferencias entre pacientes intervenidos respecto a controles, excepto en un estudio ${ }^{19}$ donde se reportó un aumento estadísticamente significativo de la calcemia en los pacientes tratados, sin llegar a hipercalcemia ni reportar otras consecuencias. El estudio restante ${ }^{24}$ evaluó la relación calciuria/ creatininuria para estimar el riesgo de nefrolitiasis, sin reportarse cambios en sus valores o eventos adversos. Otros eventos adversos como cefalea o molestias abdominales no presentaron diferencias en incidencia entre el grupo tratado respecto al control ${ }^{22}$.

\section{Discusión}

No hubo diferencias estadísticamente significativas en los desenlaces evaluados tras la suplementación con VD. Sin embargo, se recomienda interpretar de manera cautelosa los resultados finales, pues existen diversos factores que pueden haber influido en ellos. 


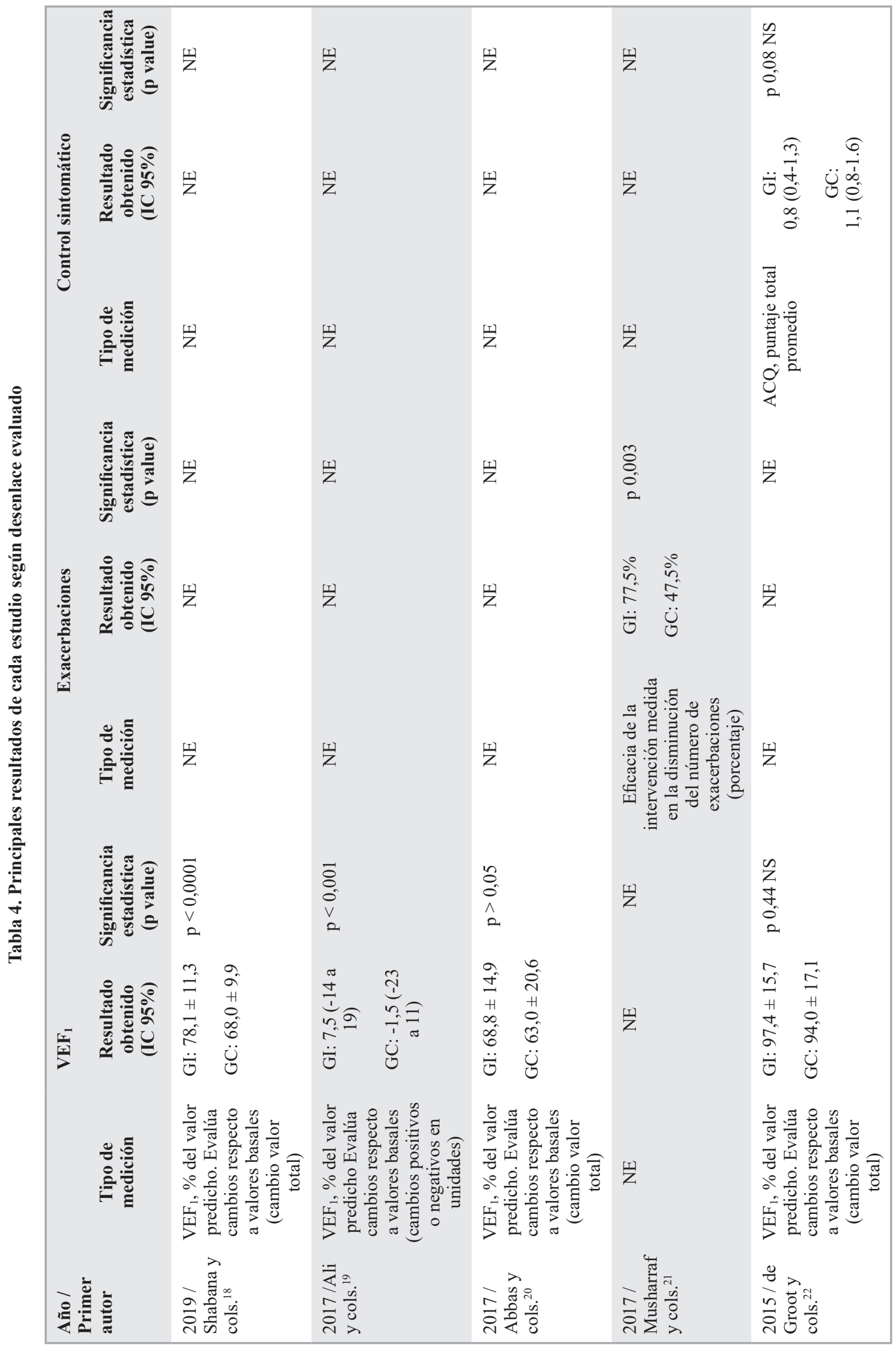




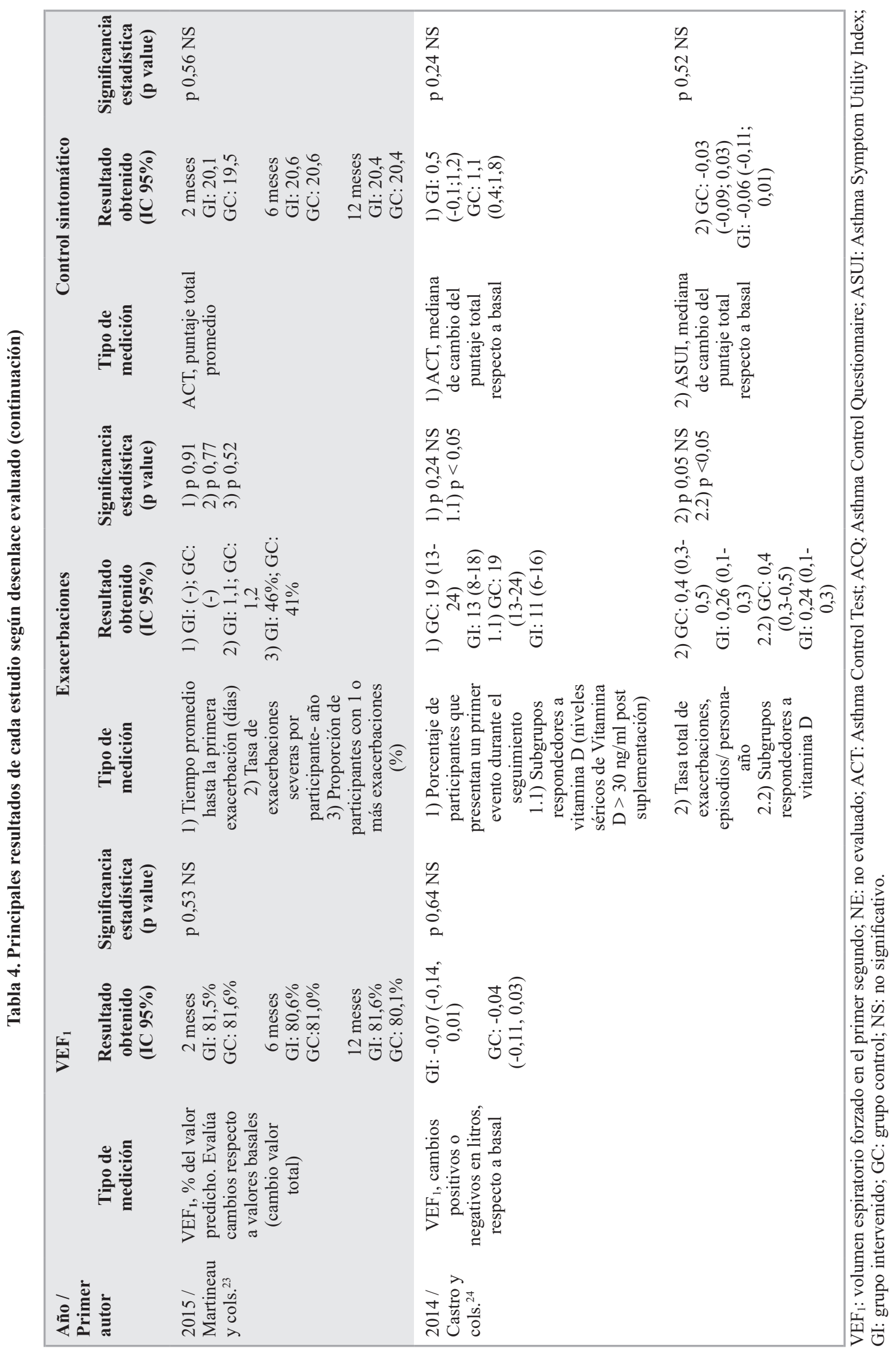


Dada la heterogeneidad de las definiciones de asma, su severidad y desenlaces clínicos evaluados, sumado a la diversidad en los resultados presentados, los autores decidieron no realizar un meta-análisis en el presente artículo, lo que impide una evaluación cuantitativa del impacto de las intervenciones y la comparación entre poblaciones, revelando la importancia de que estudios futuros se ciñan a estándares predefinidos ${ }^{25}$.

Asimismo, la diversidad de las intervenciones respecto a dosis, vía de administración, duración y metas de tratamiento, implican un sesgo de desarrollo, siendo necesaria la confección de un protocolo que permita estandarizar la suplementación de los pacientes. Se ha sugerido que un esquema de suplementación de dosis fija de VD sería menos efectivo que uno ajustado según niveles de $25(\mathrm{OH}) \mathrm{D}$ séricos ${ }^{26}$.

Las investigaciones que utilizan esquemas de suplementación con VD suelen lidiar con el problema de la variación estacional del aporte exógeno dado por la exposición cutánea diferencial a la radiación solar, pudiendo resolverse cuantificando la melanina en zonas expuestas de la pie ${ }^{24} \mathrm{o}$ diseñando estudios que permitan el seguimiento en una estación climática determinada o durante un año completo ${ }^{26}$.

Respecto a la limitante del tamaño de las cohortes, sólo dos estudios de la revisión contaron con una magnitud relevante ${ }^{23,24}$. El primero de ellos posee poca validez externa, dado su esquema escalonado de tratamiento con corticoides inhalados; el segundo estudio, por su parte, tiene menor validez interna, dada la ausencia de estandarización del tratamiento en el grupo control. Esto revela la necesidad de elaborar estudios clínicos bien diseñados para asegurar resultados óptimos y concluyentes para la aplicación clínica.

Nuestras conclusiones son concordantes con las obtenidas en el meta-análisis de Luo y cols. ${ }^{27}$, donde la suplementación con VD como tratamiento adyuvante en la población asmática adulta y pediátrica no demostró disminuir las exacerbaciones, ni mejorar la función pulmonar ni los síntomas atribuibles al asma. Contrariamente, dos meta-análisis evaluaron la eficacia de la administración de VD en pacientes asmáticos adultos y pediátricos respecto a la reducción de las exacerbaciones, uno de ellos concluyó que la VD podría reducir el riesgo de exacerbaciones asmáticas severas ${ }^{28}$, mientras que el otro evidenció un menor riesgo de exacerbaciones asmáticas ${ }^{29}$. Ambos no demostraron impacto en el control clínico y la función pulmonar.

La disparidad de estos resultados puede deberse a diferencias metodológicas con esta revisión: distintas definiciones de exacerbación asmática, inclusión de población pediátrica en ambos metaanálisis y no exclusión de pacientes con otras comorbilidades respiratorias. Además, aún no se conoce completamente la mediación por VD en procesos inflamatorios a nivel celular y molecular relacionados con la patogenia del asma, información que podría esclarecer si la suplementación favorece mayormente a ciertos fenotipos asmáticos, grupos etarios o modula diferencialmente en desenlaces clínicos como exacerbaciones, infecciones virales, función pulmonar, entre otros.

Aunque la calidad de la evidencia es muy baja para demostrar los beneficios de la VD en pacientes asmáticos, el uso del esquema de suplementación no reportó efectos adversos serios, es sencillo de implementar y es útil en la prevención y manejo de otras morbilidades ${ }^{30}$. Finalmente, no se puede descartar ni sugerir de manera directa la suplementación con VD como terapia auxiliar en pacientes asmáticos con déficit de este nutriente, dadas las conclusiones arrojadas por esta revisión. Estas recomendaciones son menos claras para quienes no presentan déficit de VD, dados los escasos estudios que han evaluado este subgrupo, haciéndose necesario un mayor estudio de esta población.

\section{Bibliografía}

1.- GLOBAL ASTHMA NETWORK. The Global Asthma Report 2018 [Internet]. Auckland, New Zealand: 2018 [consultado el 10 de agosto 2020]. Disponible en: http:// www.globalasthmanetwork.org.

2.- SEARS M. Trends in the prevalence of asthma. Chest. 2014; 145 (2): 219-25.

3.- Global Initiative for Asthma. Global strategy for asthma management and prevention: 2020 [consultado el 10 de agosto 2020]. Disponible en: www.aststhma.org

4.- HOLT P, STRICKLAND D. Interactions between innate and adaptive immunity in asthma pathogenesis: New perspectives from studies on acute exacerbations. J Allergy Clin Immunol. 2010; 125: 963-72.

5.- QUIRT J, HILDEBRAND K, MAZZA J, NOYA F, KIM H. Asthma. Allergy Asthma Clin Immunol. 2018; 14 (2): 50

6.- CAMINATI M, PHAM D, BAGNASCO D, CANONICA G. Type 2 immunity in asthma. World Allergy Organ J. 2018; 11 (1): 13.

7.- HINKS T, ZHOU X, STAPLES K, DIMITROV B, MANTA A, PETROSSIAN T, et al. Innate and adaptive $T$ cells in asthmatic patients: Relationship to severity and disease mechanisms. J Allergy Clin Immunol. 2015; 136 (2): 323-33.

8.- SASSI F, TAMONE C, D'AMELIO P. Vitamin D: 
Nutrient, Hormone, and Immunomodulator. Nutrients. 2018; 10 (11): 1656.

9.- BIZZARO G, ANTICO A, FORTUNATO A, BIZZARO N. Vitamin D and Autoimmune Diseases: ¿Is Vitamin D Receptor (VDR) Polymorphism the Culprit? Isr Med Assoc J. 2017 Jul; 19 (7): 438-43.

10.- HALL S, FISCHER K, AGRAWAL D. The impact of vitamin D on asthmatic human airway smooth muscle. Expert Rev Respir Med. 2016; 10 (2): 127-35.

11.- HALL S, AGRAWAL D. Vitamin D and Bronchial Asthma: An Overview of Data From the Past 5 Years. Clin Ther. 2017; 39 (5): 917-29.

12.- BOZZETTO S, CARRARO S, GIORDANO G, BONER A, BARALDI E. Asthma, allergy, and respiratory infections: the vitamin D hypothesis. Allergy 2012; 67: 10-17.

13.- HEANEY R, HOLICK M. Why the IOM recommendations for vitamin D are deficient. J Bone Miner Res. 2011; 26: 455-7.

14.- CASSIM R, RUSSELL M, LODGE C, LOWE A, KOPLIN J, DHARMAGE S. The role of circulating 25 hydroxyvitamin D in asthma: a systematic review. Allergy 2015; 70: 339-54.

15.- BREHM J, SCHUEMANN B, FUHLBRIGGE A, HOLLIS B, STRUNK R, ZEIGER R, et al. Serum vitamin $\mathrm{D}$ levels and severe asthma exacerbations in the Childhood Asthma Management Program study. J Allergy Clin Immunol. 2010; 126 (1): 52-58e5.

16.- THUESEN B, SKAABY T, HUSEMOEN L, FENGER M, JØRGENSEN T, LINNEBERG A. The association of serum $25-\mathrm{OH}$ vitamin D with atopy, asthma, and lung function in a prospective study of Danish adults. Clin Exp Allergy. 2015; 45 (1): 265-72.

17.- BRUMPTON B, LANGHAMMER A, HENRIKSEN A, CAMARGO C, CHEN Y, ROMUNDSTAD P, et al. Vitamin D and Lung Function Decline in Adults With Asthma: The HUNT Study. Am J Epidemiol. 2016; 183 (8): 739-46.

18.- SHABANA M, ESAWY M, ISMAIL N, SAID A. Predictive role of IL-17A/IL-10 ratio in persistent asthmatic patients on vitamin D supplement. Immunobiology. 2019; 224 (6): 721-7.

19.- ALI A, SELIM S, ABBASSI M, SABRY N. Effect of alfacalcidol on the pulmonary function of adult asthmatic patients: A randomized trial. Ann. Allergy Asthma Immunol. 2017; 118: 557-63.

20.- ABBAS R, ABDULRIDHA M, SHAFEK M. Clinical evaluation of potential anti-inflamatory effect of vitamin D3 adjuvant therapy for chronic asthma in iraqi patients. Int J Pharm Pharm Sci. 2016; 9: 139-44.

21.- MUSHARRAF M, SANDHU G, MUMTAZ M, RASHID M. Role of vitamin D in prevention of acute exacerbation of bronchial asthma in adults. J Postgrad Med Inst. 2017; 31 (3): 310-3.

22.- DE GROOT J, VAN ROON E, STORM H, VEEGER N, ZWINDERMAN A, HIEMSTRA P, et al. Vitamin $D$ reduces eosinophilic airway inflammation in nonatopic asthma. J Allergy Clin Immunol. 2015; 135 (3): 670-5e3.

23.- MARTINEAU A, MACLAUGHLIN B, HOOPER R, BARNES N, JOLLIFFE D, GREILLER C, et al. Double-blind randomised placebo-controlled trial of bolus-dose vitamin D3 supplementation in adults with asthma (ViDiAs) Thorax. 2015; 0: 1-7.

24.- CASTRO M, KING T, KUNSELMAN S, CABANA M, DENLINGER N, HOLGUIN F, et al. Effect of Vitamin $\mathrm{D}_{3}$ on Asthma Treatment Failures in Adults With Symptomatic Asthma and Lower Vitamin D Levels: The VIDA Randomized Clinical Trial. JAMA. 2014; 311 (20): 2083-91.

25.- REDDEL H, TAYLOR D, BATEMAN E, BOULET L, BOUSHEY F, BUSSE W, et al. An official American Thoracic Society/European Respiratory Society statement: asthma control and exacerbations: standardizing endpoints for clinical asthma trials and clinical practice. Am J Respir Crit Care Med. 2009; 180 (1): 59-99.

26.- BREHM J. Vitamin D and asthma-life after VIDA? Curr Allergy Asthma Rep. 2014; 14 (9): 461.

27.- LUO J, LIU D, LIU C. Can Vitamin D Supplementation in Addition to Asthma Controllers Improve Clinical Outcomes in Patients With Asthma?: A Meta-Analysis. Medicine (Baltimore). 2015; 94 (50): e2185.

28.- MARTINEAU A, CATES C, URASHIMA M, JENSEN M, GRIFFITHS A, NURMATOV U, et al. Vitamin D for the management of asthma. Cochrane Database Syst Rev. 2016; 9: CD011511.

29.- WANG M, LIU M, WANG C, XIAO Y, AN T, ZOU $\mathrm{M}$, et al. Association between vitamin $\mathrm{D}$ status and asthma control: A meta-analysis of randomized trials. Respiratory Medicine 2019; 150: 85-94.

30.- GIL Á, PLAZA-DIAZ J, MESA M. Vitamin D: Classic and Novel Actions. Ann Nutr Metab. 2018; 72 (2): 87-95.

Correspondencia a:

Demian Martínez Pardo

Facultad de Medicina, Universidad de Chile.

Av. Independencia 1027, Independencia.

Santiago, Chile.

Email: demianchi@ug.uchile.cl 